\section{Bei Morbus Bechterew werden Brüche der Wirbelsäule oft übersehen}

Wirbelsäulenfrakturen werden bei Patienten mit ankylosierender Spondylitis (AS, Morbus Bechterew) initial oft unterschätzt oder gar übersehen. Dabei besteht bei Betroffenen die Gefahr schwerer neurologischer Schäden.

$B^{\mathrm{c}}$ i der Analyse von 129 operativ versorgten Wirbelsäulenfrakturen bei AS-Patienten haben Dr. Manuel Backhaus vom BG-Universitätsklinikum in Bochum und seine Kollegen festgestellt, dass die Hälfte der Stürze eine inkomplette oder komplette Querschnittsymptomatik ausgelöst hatte. In der bislang nach eigenen Angaben bislang größten in der Literatur veröffentlichten Serie waren $28 \%$ der Frakturen zunächst übersehen oder als stabil eingeschätzt worden. „Eine konventionelle radiologische Diagnostik genügt häufig nicht", betonen die Chirurgen und Neurotraumatologen.
Goldstandard sei die Computertomografie (CT), die in besonderen Fällen noch um eine Magnetresonanztomografie (MRT) ergänzt werden sollte. Denn die veränderte Biomechanik der Wirbelsäule mit verminderten Kompensationsmechanismen verursache infolge der Instabilität überdurchschnittlich oft neurologische Symptome, zumal ja auch die verknöcherten Bänder und Gewebe verletzt werden.

Insgesamt waren unter den Verletzten 51 Patienten mit HWS-Frakturen, 55 mit BWS- und 23 Patienten mit LWS-Frakturen. In $44 \%$ der Fälle war anamnestisch lediglich ein banales Trauma in der

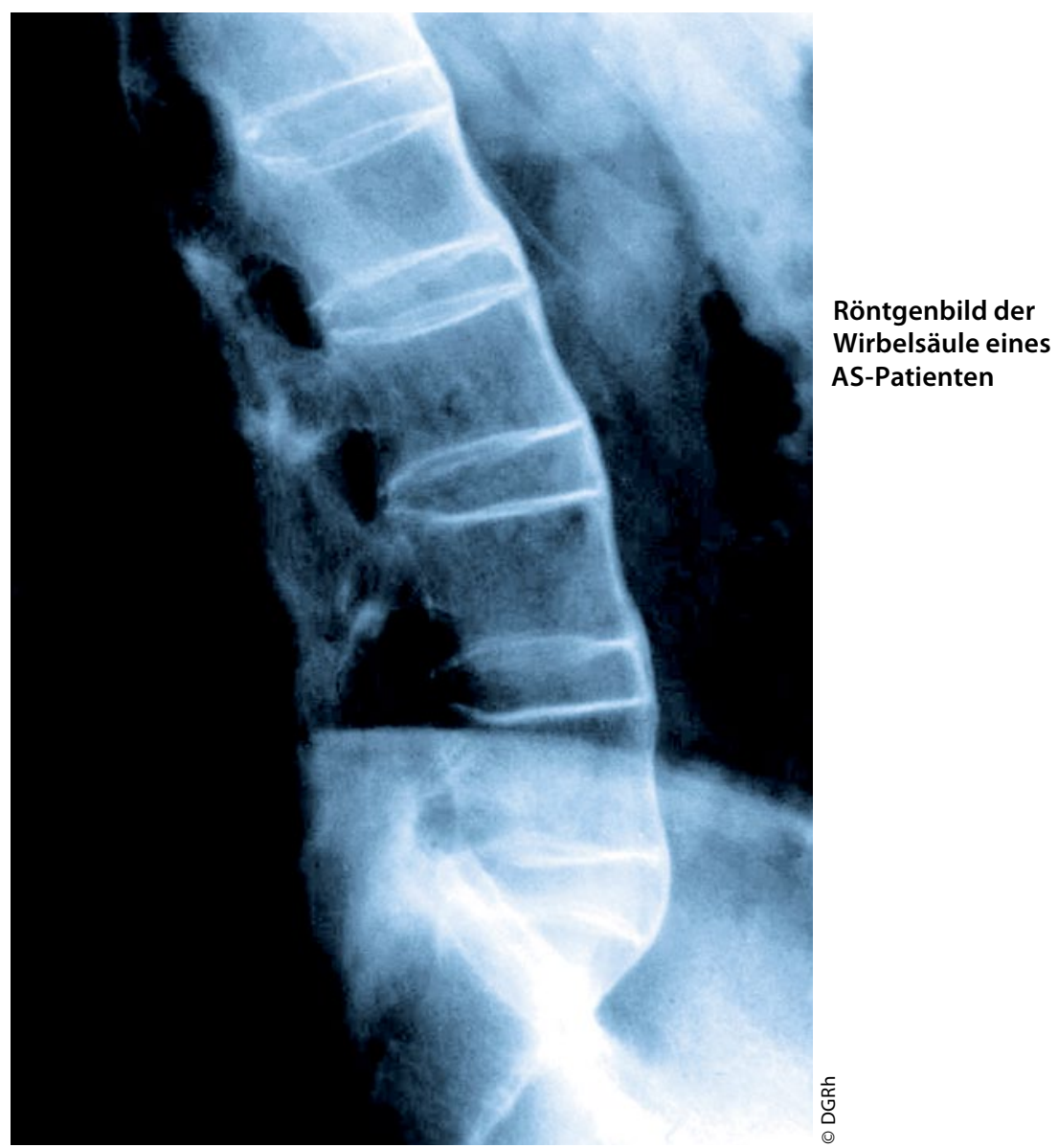

häuslichen Umgebung voraugegangen, bei $45 \%$ der Patienten ein adäquates Trauma.

Schlussfolgerung: Die Bochumer Mediziner halten bei AS-Patienten mit Wirbelsäulenfrakturen wegen der oft hochgradigen Instabilität die operative Versorgung mit langstreckigen Spondylodesen für zwingend erforderlich. Allerdings muss mit Komplikationen gerechnet werden, nicht zuletzt wegen häufiger Komorbiditäten. So kam es bei $14 \%$ der Patienten zu einem postoperativen Wundinfekt, bei $15 \%$ lockerten sich die Implantate oder die Stabilisierung war unzureichend. $27 \mathrm{der}$ im $\mathrm{Me}$ dian 73 Jahre alten Patienten (23\%) starben während des stationären Aufenthalts, davon $13 \%$ infolge postoperativer Komplikationen wie Multiorganversagen, Sepsis und Exazerbation von Vorerkrankungen. Dr. Thomas Meißner

Kommentar: Es ist bekannt, dass Patienten mit ankylosierender Spondylitis ein höheres Risiko tragen, bei Bagatelltraumata Wirbelsäulenfrakturen zu erleiden. Wie in der Arbeit gefordert, sollte bei allen ASPatienten nach einem Sturz auf die Wirbelsäule eine Computer- oder eine Magnetresonanztomografie durchgeführt werden. Damit können spätere Komplikation umgangen werden. Mit der CT wurde eine hohe Anzahl primär nicht erkannter Frakturen nachgewiesen. Bemerkenswert oft fanden die Autoren auch Querschnittverletzungen. Die von den Autoren empfohlene zwingende operative Versorgung einer Querschnittverletzung sollte im Einzelfall entschieden werden. Auf jeden Fall erfordert die Feststellung einer Fraktur eine besondere Form der Behandlung.

Backhaus, M et al.: Wirbelsäulenfraktur bei ankylosierender Spondylitis. Orthopäde 2011, 40(10): 917-924

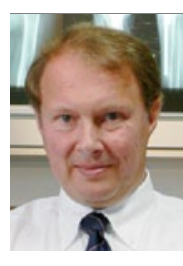

Prof. Dr. med. habil. Marc Thomsen

Chefarzt Orthopädie und Unfallchirugie, spezielle orthopädische Chirurgie und Rheumatologie DRK-Klinik Baden-Baden Lilienmattstr. 5 76530 Baden-Baden 